\title{
Densitometria ossea: una breve guida per il nefrologo
}

Giornale di Tecniche Nefrologiche e Dialitiche 2019, Vol. 3I(2) 89-93

(C) The Author(s) 2019

Article reuse guidelines:

sagepub.com/journals-permissions

DOI: 10.1 | 177/03949362। 9846435

journals.sagepub.com/home/gtn

\section{(3)AGE}

\author{
Francesco lannuzzella', Giulio Pioli², Mariarosa Maiorana' \\ e Mariacristina Gregorini'
}

\begin{abstract}
DXA: a beginner's guide

Studies in patients with chronic kidney disease have definitively shown that "dual-energy X-ray absorptiometry" (DXA) may be helpful in assessing fracture risk. Moreover, it is essential to diagnose osteoporosis and evaluate response to treatment. In order to obtain the best utility from DXA, nephrologists need to know the fundamentals of this technique, its indications in clinical practice, and the potential pitfalls in the interpretation of its results. This review will provide an overview of the fundamentals, methodology, and current clinical applications of DXA with special focus on the nephrologist's perspective.
\end{abstract}

\section{Keywords}

DXA, MOC, BMD, Bone quality, Bone density, Osteoporosis, CKD-MBD

\section{Introduzione}

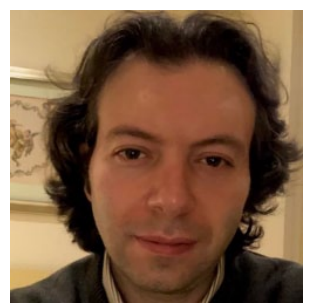

Si stima che nei pazienti con malattia renale cronica le fratture presentino un'incidenza da 2 a 14 volte più elevata rispetto alla popolazione generale. ${ }^{1,2}$ Per i pazienti in dialisi $\mathrm{i}$ dati disponibili sono quelli della coorte DOPPS, comprendente 34,597 emodializzati osservati nel periodo 1996-2011. In questo studio, il 3\% presentava un evento fratturativo, con un'incidenza in Italia di quasi 20 eventi/1,000 pazienti-anno. Tra i pazienti portatori di trapianto renale, il rischio di frattura del femore risulta addirittura maggiore del $34 \%$ rispetto ai pazienti dializzati $^{2}$ e da 2 a 23 volte più elevato rispetto alla popolazione generale. Nei soggetti con malattia renale cronica, le fratture da fragilità mostrano poi caratteristiche del tutto peculiari: in particolare, si presentano in genere in soggetti di 10-15 anni più giovani rispetto al resto della popolazione ${ }^{3}$ esi caratterizzano per tassi di ospedalizzazione e mortalità di gran lunga più elevati con effetti rilevanti sulla spesa sanitaria. ${ }^{4}$

I meccanismi fisiopatologici alla base di questo aumento così consistente del rischio fratturativo nella popolazione affetta da malattia renale cronica sono complessi e non ancora del tutto chiariti. Oltre al disordine del metabolismo minerale correlato alla malattia renale cronica (la cosiddetta CKD-MBD, Chronic Kidney Disease-related to Mineral Bone Disorders), vari fattori sembrano concorrere nel determinare, in gradi e forme variabili, una riduzione della resistenza ossea. ${ }^{5}$ Quest'ultima, più nel dettaglio, è il prodotto di due determinanti fondamentali per la salute dell'osso: la densità minerale ossea (Bone Mineral Density, BMD) e la qualità ossea (Bone Quality, BQ). La BQ è costituita da un insieme di più componenti strutturali e metaboliche quali la microarchitettura, il turnover, la mineralizzazione, l'accumulo di microdanni. La BMD esprime invece la quantità di minerale per area ossea $\left(\mathrm{g} / \mathrm{cm}^{2}\right)$, un parametro comunemente utilizzato per la predizione del rischio di frattura.

Sebbene siano stati ideati e testati in ambito di ricerca diversi strumenti alternativi per la quantificazione della $\mathrm{BQ}$, questi sono ancora lontani dall'applicazione pratica. La mineralometria (absorptiometria) ossea è tuttora lo

\footnotetext{
'Struttura Complessa di Nefrologia e Dialisi, Azienda USL-IRCCS di Reggio Emilia, Italy

${ }^{2}$ Ortogeriatria, Azienda USL-IRCCS di Reggio Emilia, Italy

\section{Corrispondenza:}

Dr. Francesco lannuzzella, SC di Nefrologia e Dialisi, Arcispedale S.

Maria Nuova, Viale Risorgimento 80, 42123 Reggio Emilia, Italy.

E-mail: proarchia@yahoo.it
} 
strumento di più largo impiego e quello che offre i risultati di maggiore valore pratico in ambito clinico. La "dualenergy X-ray absorptiometry" (DXA), la metodica di mineralometria oggi più diffusa, è perlopiù utilizzata per la quantificazione della $\mathrm{BMD}$, la cui utilità nella predizione del rischio fratturativo è ora ampiamente accettata anche nella malattia renale cronica di stadi 3-5D. ${ }^{6}$

In questa revisione, verranno discusse le caratteristiche tecniche, l'interpretazione e le applicazioni cliniche della DXA con particolare riferimento all'ambito nefrologico.

\section{La mineralometria}

\section{Presupposti tecnologici}

La DXA utilizza raggi $\mathrm{X}$ ad alta e bassa energia fotonica. Il principio alla base della DXA è rappresentato infatti dalla differenza nell'assorbimento dei fotoni tra i tessuti molli e le ossa. ${ }^{7-9}$ I tessuti più densi e più spessi consentono a un numero minore di fotoni di passare attraverso il rivelatore rispetto a quelli a minore densità. La differenza nell'assorbimento di questi fasci distingue l'osso dai tessuti molli e consente la quantificazione della BMD. Ai fini diagnostici e di predizione del rischio fratturativo, la $\mathrm{BMD}$ rilevata viene quindi confrontata con una popolazione di riferimento.

A seconda del dispositivo utilizzato, i fotoni possono essere ottenuti utilizzando due meccanismi. In alcuni casi, il generatore emette radiazioni alternate di alto (140 $\mathrm{kVp}$ ) e basso (70-100 kVp) kilovoltaggio mentre si sposta sulla superficie del corpo da esaminare. In altri, il generatore emette un raggio costante poi separato attraverso un filtro in fotoni ad alta $(70 \mathrm{keV})$ e bassa energia $(40 \mathrm{keV})$. I sistemi DXA disponibili includono diversi tipi di hardware (filtri, collimatori, rivelatori) e software (algoritmi di analisi). ${ }^{8}$ La sorgente di raggi X può utilizzare un collimatore tipo pinhole, nel quale l'assorbimento è registrato da un singolo rivelatore, o un collimatore a fessura, nel quale l'assorbimento è invece registrato da un rilevatore multiplo. Quest'ultimo sistema, in particolare, riduce il tempo di acquisizione e migliora la qualità dell'immagine. Allo stesso tempo, l'algoritmo di analisi discrimina l'osso dai tessuti molli in un modo variabile.

Le principali modalità DXA utilizzate nella pratica clinica sono la densitometria assiale ossea, l'opzione di scelta per misurare la BMD, e la densitometria dell'intero corpo, usata per valutare la composizione corporea. ${ }^{10}$ Quest'ultima è particolarmente utile nei pazienti con disordini del peso secondari a malattie endocrine e in pazienti pediatrici con crescita ritardata, ma può essere utile anche per valutare la lipodistrofia associata a infezioni retrovirali, nel monitoraggio delle artroplastiche o per determinare il rischio cardiovascolare. In particolare, in ambito nefrologico, è stata adottata per la validazione di diversi bioimpedenziometri.
Sebbene altre tecniche di imaging siano potenzialmente utili per valutare e misurare la struttura ossea e studiare la $\mathrm{BQ}$, la DXA assiale della colonna lombare e dell'anca, cosiddetta DXA centrale, è attualmente la tecnica di scelta per studiare l'osteoporosi. Il fatto che siano disponibili in commercio dispositivi (Lunar, Hologic, Norland) con caratteristiche spesso molto diverse fra loro ha delle implicazioni pratiche. Essendo difficile la comparazione dei dati acquisiti nel tempo su dispositivi diversi, è infatti consigliabile eseguire il follow-up di ogni paziente sempre con lo stesso dispositivo. È noto in effetti che l'accuratezza della densitometria ossea eseguita sempre sul medesimo sistema DXA è alta con un margine di errore dell'ordine di $1 \%-2 \%$.

\section{Indicazioni}

Nel 1994, l'OMS ha introdotto la misurazione della BMD con DXA come standard di riferimento per quantificare l'osteoporosi. ${ }^{11}$

Le principali indicazioni DXA per lo studio della BMD sono riportate in tabella 1. Uno studio DXA finalizzato alla valutazione della BMD è stato riportato dagli autori Albanese et al. ${ }^{10}$ e da Lewiecki et al. ${ }^{11}$ :

- Pazienti di età $\geqslant 50$ anni con una frattura non vertebrale recente o considerati ad alto rischio per fratture. I pazienti di pari età con una frattura vertebrale comprovata possono anch'essi trarre beneficio da una DXA.

- Pazienti di età $\geqslant 60$ anni e senza storia di recente frattura ma ad alto rischio di fratture.

- Pazienti con condizione o farmaco sottostante associati a possibile perdita ossea.

- Follow-up durante trattamento anti-riassorbitivo o dopo i 2 anni di terapia anabolica con teripatide/ PTH (1-84). Il follow-up può essere utile anche 2-3 anni dopo la cessazione del trattamento.

La DXA è indicata nella valutazione della composizione corporea totale ${ }^{10}$ in pazienti con:

- HIV: per valutare la distribuzione del grasso nei pazienti che usano farmaci antiretrovirali associati ad un rischio di lipo-atrofia

- Obesità sottoposta a chirurgia bariatrica soprattutto in caso di perdita di peso elevata.

Le controindicazioni alla DXA comprendono gravidanza in atto, recente $(<5$ giorni) somministrazione orale di un mezzo di contrasto e la recente esecuzione di uno studio isotopico ( $<2$ giorni). I mezzi di contrasto, in particolare, possono determinare sovrastima densitometrica e per lo stesso motivo è consigliabile la sospensione preliminare alcuni giorni prima dell'esame dei chelanti a base di lantanio. Questi chelanti infatti, unici fra quelli attualmente 
Tabella I. Indicazioni alla BMD.

\begin{tabular}{ll}
\hline Donne & Tutte le donne di età $\geqslant 65$ anni per screening asintomatico \\
& Tutte le donne in post-menopausa di età $<65$ anni e gli uomini di età $<70$ anni che \\
& presentano fattori di rischio per l'osteoporosi \\
\hline Uomini & - Uomini di età $\geqslant 70$ anni \\
& - Uomini di età $<70$ anni con fattori di rischio per osteoporosi \\
Indipendentemente dal sesso & Maschi ipogonadici postpuberali con castrazione chirurgica o chemioterapica \\
& - Individui di qualsiasi età con risultati indicativi di demineralizzazione o fratture da fragilità \\
& - Monitoraggio della terapia antiosteoporotica \\
& - Soggetti con malattie associate a riduzione della BMD (inclusa l'insufficienza renale cronica) \\
& - Individui che iniziano o ricevono terapia a lungo termine con farmaci noti per influenzare \\
& negativamente la BMD \\
& Soggetti con un disturbo endocrino noto per influire negativamente sulla BMD (ad es. \\
& iperparatiroidismo, ipertiroidismo o sindrome di Cushing)
\end{tabular}

in uso, se non correttamente assunti, possono lasciare residui radio-opachi nelle anse intestinali che si sovrappongono nelle immagini DXA alla proiezione dei corpi vertebrali.

\section{Aree di studio}

Per la maggior parte dei pazienti adulti, l'esame DXA dovrebbe includere la colonna lombare e il femore prossimale; l'avambraccio può essere utilizzato in sostituzione quando misurazioni all'anca o alla colonna vertebrale non possono essere ottenute. ${ }^{12}$ Nei bambini e adolescenti (soggetti di età inferiore ai 20 anni), la misurazione viene eseguita solo nella colonna lombare. ${ }^{13}$

In ciascuna scansione viene selezionata una regione di interesse (ROI) standardizzata in base al tipo di dispositivo che dovrà essere sempre sovrapponibile alla precedente negli esami utilizzati per il follow-up. Dopo aver definito la ROI, il contenuto minerale osseo (BMC) viene infatti calcolato dal computer e sarà correlato all'area misurata per ottenere il valore finale di BMD. Sebbene i sistemi DXA propongano automaticamente la selezione delle ROI, sia il tecnico che il radiologo devono convalidarli e, a seconda del caso, correggerli. ${ }^{14}$

La scansione postero-anteriore (PA) della colonna lombare include i corpi vertebrali da L1 a L4. Per la corretta selezione della ROI, va ricordato che, sebbene alcuni soggetti presentino coste sovrannumerarie, D12 è solitamente l'ultima vertebra connessa ad una costa, e che L3 di solito è la vertebra con il processo trasversale più lungo. Dopo aver correttamente selezionato la ROI, un valore finale di BMD è quindi ottenuto da una media di tutte quattro le vertebre selezionate, a meno che non ci sia una grave interferenza per esempio a causa di una marcata osteoartrosi, di calcificazioni vascolari a carico dell'aorta addominale o dopo un intervento chirurgico radicale con o senza materiale protesico interposto. In questi casi infatti come pure nell'eventualità in cui alcune delle vertebre selezionate presentino cambiamenti strutturali o artefatti dovuti a fratture o a lesioni focali, la misurazione della BMD sarà da limitare alle sole vertebre valutabili per limitare il rischio di un'interferenza sulla rilevazione densitometrica. ${ }^{15}$ Per lo stesso motivo, sarà da escludere una vertebra che presenti una differenza di T-score $>1.0$ rispetto alle vertebre adiacenti. In ogni caso, per misurare la BMD alla colonna lombare, sono necessarie almeno due vertebre valutabili.

Lo studio del femore può essere eseguito indistintamente all'anca destra o sinistra, anche se è opportuno ripetere lo studio di follow-up sempre dalla stessa parte. I femori con alterazioni dovute a frattura, lesioni focali o sostituzioni protesiche saranno anch'essi ovviamente da escludere. Il valore di BMD da prendere a riferimento è dato dal valore più basso tra quello del femore prossimale totale e quello del collo del femore.

Come già detto, lo studio dell'avambraccio non dominante è effettuato per avere una seconda regione misurabile quando l'anca o la colonna vertebrale non possono essere misurate, e spesso nei pazienti grandi obesi per far fronte a difficoltà tecniche. L'avambraccio è inoltre l'area di studio preferenziale per la misurazione della BMD nei pazienti affetti da iperparatiroidismo primitivo poiché la densità delle ossa dell'avambraccio cambia più precocemente di quella dello scheletro assile.

\section{Modalità di esecuzione}

Il paziente non richiede alcuna preparazione specifica ad eccezione della rimozione di oggetti metallici situati nell'area del corpo indagata. Nei pazienti in trattamento con chelanti del fosforo a base di lantanio, se ne consiglia la sospensione perché eventuali residui a livello intestinale potrebbero sovrapporsi proiettivamente all'area in esame, determinando una sovrastima della BMD a livello lombare. 
Tabella 2. Alcuni termini comunemente utilizzati in densitometria ossea.

\begin{tabular}{ll}
\hline BMC & Contenuto minerale osseo \\
BMD & Densità minerale ossea \\
T-score & Differenza nel numero di deviazioni standard tra il valore medio della \\
& BMD del paziente e la media di una popolazione di riferimento \\
Z-score & Differenza nel numero di deviazioni standard tra il valore medio della \\
& BMD del paziente e la media di una popolazione di riferimento della \\
& stessa razza, sesso e età. \\
Osteopenia & T-score tra - I e -2.5 \\
Osteoporosi & T-score $\leqslant-2.5$ \\
Normale massa ossea & T score $>-I$ \\
Massa ossea inferiore a quanto atteso per l'età & Z-score $<-2$. Lo Z-score è utilizzato nelle donne in premenopausa, negli \\
& uomini di età $<50$ anni, nei bambini e negli adolescenti \\
\hline
\end{tabular}

\section{Posizionamento del paziente}

L'ottimizzazione della posizione del paziente sul lettino della DXA è essenziale. Il posizionamento errato è una delle cause principali di errori nella stima della BMD. Nello studio PA della colonna lombare, il paziente giace supino sul tavolo con le gambe flesse a $90^{\circ}$ su un grosso cuscino di supporto a forma di cubo che riduce la lordosi lombare avvicinando la colonna vertebrale al tavolo.

Per lo studio dell'anca, il paziente giace supino con le gambe leggermente in abduzione per mantenere l'asse femorale diritto e con una lieve rotazione interna $\left(15^{\circ}\right.$ $30^{\circ}$ ). Nello studio dell'anca, la ROI deve essere collocata al collo del femore, evitando la sovrapposizione del ramo ischiopubico e del grande trocantere. La corretta posizione sarà confermata dal fatto che il piccolo trocantere non sia visibile sull'immagine analizzata.

Nello studio dell'avambraccio, il paziente è seduto di fianco al tavolo con l'avambraccio appoggiato su di esso conla mano in pronazione etenutain sede. Nell'avambraccio, l'area di analisi è impostata sul radio distale, con la linea di riferimento nel processo stiloideo.

Indipendentemente dalla sede, l'immagine acquisita deve includere 1-2 cm sopra e sotto l'area da riprendere, con l'osso da analizzare dritto e ben centrato.

Per quanto riguarda il tempo di scansione, i primi densitometri con collimatori pinhole richiedevano circa 5 minuti per scansione, ma con i dispositivi più recenti le immagini possono essere ottenute in meno di un minuto.

\section{Interpretazione}

Parametri valutati in densitometria osseal6. Dopo l'acquisizione e l'analisi, il software del sistema DXA calcola vari parametri (tabella 2). Il BMC è la quantità di calcio stimata dall'energia assorbita in una regione specifica. La BMD, molto più rilevante, è la quantità media di minerali per unità di superficie, ed è pertanto calcolata dividendo il BMC per unità di superficie $\left(\mathrm{g} / \mathrm{cm}^{2}\right)$. I valori usati per la diagnosi ( $\mathrm{T}$ e $\mathrm{Z}$ score) si ottengono confrontando $i$ dati rilevati con un database di riferimento. Le linee guida consigliano di utilizzare preferibilmente il database NHANES III come riferimento standard per il collo del femore e dell'anca totale e utilizzare invece il database del produttore per la colonna lombare.

Il T-score è il valore utilizzato per diagnosticare l'osteoporosi nelle donne in postmenopausa e negli uomini di età pari o superiore a 50 anni. Secondo le definizioni OMS e ISCD, il T-score è il numero di deviazioni standard della BMD del paziente sopra o sotto la media per la popolazione di riferimento del NHANES III costituita da un gruppo uniforme di giovani donne caucasiche adulte. Secondo questi criteri, viene considerato normale un T-score $>-1.0$, mentre definisce una situazione di osteopenia un T-score tra - 1 e - 2.5 , e una di osteoporosi un T-score $<-2.5$. È tuttavia da sottolineare che molti sistemi DXA in uso utilizzano database propri diversi in base al sesso.

Lo Z-score è invece utilizzato nelle donne in premenopausa e negli uomini di età inferiore ai 50 anni, nonché nei bambini e negli adolescenti. Lo Z-score è il numero di deviazioni standard della BMD del paziente sopra o sotto la media per una popolazione di riferimento della stessa razza, sesso ed età. Uno Z-score $<-2$ identifica i soggetti con una massa ossea "inferiore all'intervallo previsto per l'età".

Il follow-up strumentale con DXA dovrebbe essere eseguito solo quando si prevedono cambiamenti nella BMD superiori al minimo cambiamento significativo (least significant change, LSC) del dispositivo utilizzato o quando più misure sono indicate in un periodo di tempo più lungo. L'LSC dipende dalla precisione della tecnica. Con una precisione rispettivamente dell' $1 \%$, del $2 \%$ e del $3 \%$, le LSC sono rispettivamente del $2.77 \%, 5.5 \%$ e $8.3 \%$. Va però ricordato che nei pazienti con osteoporosi la precisione è inferiore a causa della diminuzione del BMC. Quando possibili, i confronti vanno fatti prendendo a riferimento il valore assoluto della BMD (non il T-score) perché aggiornamenti sul database di riferimento correlato allo specifico software DXA potrebbero interferire con la valutazione comparativa. Inoltre, come ricordato in precedenza, il confronto quantitativo della BMD tra dispositivi diversi è assai difficile e di fatto non è possibile senza uno studio di calibrazione incrociata. 
Studio delle fratture vertebrali (VFA). Le fratture vertebrali possono risultare clinicamente silenti in circa $2 / 3$ dei pazienti. Il Vertebral Fracture Assessment (VFA) è una metodologia DXA per visualizzare fratture vertebrali. Con un'immagine laterale della colonna toracica e lombare, in modo simile agli approcci radiologici standard utilizzati per la morfometria vertebrale, è possibile effettuare lo studio VFA mediante un software specifico disponibile per la determinazione dell'altezza vertebrale. ${ }^{17}$

Il software posiziona sei punti di riferimento sul contorno di ogni vertebra misurandone le altezze anteriore, media e posteriore. Le deformità vertebrali sono determinate e classificate in base alla morfologia (a cuneo, biconcava o schiacciamento) e all'entità della riduzione di altezza: lieve $(20 \%-25 \%)$, moderata $(25 \%-40 \%)$ o grave $(>40 \%)$.

La valutazione della $B Q$ tramite $D X A$. Una stima del rischio fratturativo, indipendente dalla $\mathrm{BMD}$, può essere ottenuta dalla valutazione del Trabecular Bone Score o TBS. Si tratta di un dato che fornisce informazioni relative alla microarchitettura dell'osso ed è ricavato attraverso specifici algoritmi/software DXA a partire dall'immagine della colonna vertebrale lombare attraverso una scala di grigi. ${ }^{18}$

\section{La refertazione}

Il referto dovrebbe contenere l'indicazione per il test, i siti misurati e i relativi T e Z-score. Quando misurati, T e Z-score della colonna vertebrale lombare totale, del collo del femore e dell'anca totale vanno inclusi nel referto. Se uno o entrambi i siti non sono adatti per la misurazione o se il paziente presenta un iperparatiroidismo primario, dovrebbero essere riportati T e Z-score del terzo distale del radio.

Per la valutazione del rischio di frattura assoluta a 10 anni attraverso lo strumento FRAX, è indicato l'utilizzo della BMD del collo del femore.

La conclusione dovrebbe contenere la diagnosi in termini di BMD normale, osteopenia o osteoporosi e se presente quantità, grado e localizzazione delle fratture vertebrali. In tutti i casi in cui venga eseguita la VFA, va riportata l'assenza o la presenza di fratture vertebrali, definite come riduzione dell'altezza del corpo vertebrale superiore al $25 \%-40 \%$ o superiore al $40 \%$. Nell'eventualità di anomalie strutturali focali, è opportuno raccomandare ulteriori indagini, essendo la VFA adatta solo per rilevamento delle fratture vertebrali.

\section{Dichiarazione di assenza di conflitto di interessi}

Gli Autori dichiarano di non avere conflitti di interessi.

\section{Finanziamenti}

Gli Autori dichiarano di non aver ricevuto finanziamenti specifici da qualsiasi ente nei settori pubblico, privato o senza fini di lucro.

\section{Bibliografia}

1. Alem AM, Sherrard DJ, Gillen DL, et al. Increased risk of hip fracture among patients with end-stage renal disease. Kidney Int 2000; 58:396-399.

2. Ball AM, Gillen DL, Sherrard D, et al. Risk of hip fracture among dialysis and renal transplant recipients. JAMA 2002; 288: 3014-3018.

3. Coco M and Rush H. Increased incidence of hip fractures in dialysis patients with low serum parathyroid hormone. Am J Kidney Dis 2000; 36:1115-1121.

4. Lin JC and Liang WM. Mortality and complications after hip fracture among elderly patients undergoing hemodialysis. BMC Nephrol 2015; 16:100.

5. Kazama JJ. Chronic kidney diseases and fragility fracture. Clin Exp Nephrol 2017; 21(Suppl 1): 46-52.

6. Ketteler M, Block GA, Evenepoel P, et al. Executive summary of the 2017 KDIGO Chronic Kidney DiseaseMineral and Bone Disorder (CKDMBD) Guideline Update: what's changed and why it matters. Kidney Int 2017; 92(1):26-36. doi: 10.1016/j.kint.2017.04.006.

7. Adams JE. Single and dual energy X-ray absorptiometry. Eur Radiol 1997; 7:S20-31.

8. Genant HK. Current state of bone densitometry for osteoporosis. Radiographics 1998; 18:913-918.

9. Blake GM and Fogelman I. Technical principles of dual energy X-ray absorptiometry. Semin Nucl Med 1997; 27:210-228.

10. Albanese CV, Diessel E and Genant HK. Clinical applications of body composition measurements using DXA. J Clin Densitom 2003; 6:75-85.

11. Lewiecki EM, Watts NB, McClung MR, et al. Official positions of the International Society for Clinical Densitometry. J Clin Endocrinol Metab 2004; 89:36513655 .

12. Baim S, Binkley N, Bilezikian JP, et al. Official position of the International Society for Clinical Densitometry and executive summary of the 2007 ISCD position development conference. J Clin Densitom 2008; 11:75-91.

13. Baim S, Leonard MB, Bianchi ML, et al. Official positions of the International Society for Clinical Densitometry and executive summary of the 2007 ISCD pediatric position development conference. J Clin Densitom 2008; 11:6-21.

14. Staron RB, Greenspan R, Millar TT, Bilezikian JP, Shane E and Haramati N. Computerized bone densitometric analysis: operator-dependent errors. Radiology 1999; 211:467-470.

15. Jacobson JA, Jamadar DA and Hayes CW. Dual X-ray absorptiometry. Recognizing image artifacts and pathology. Am J Roentgenol 2000; 174:1699-1705.

16. Lorente-Ramos R, Azpeitia-Armán J, Munoz-Hernández A, García-Gómez JM, Díez-Martínez P and Grande-Bárez M. Dual-energy X-ray absorptiometry in the diagnosis of osteoporosis. A practical how-to guide. Am J Roentgenol 2011; 196:897-904.

17. Genant HK, Wu CY, van Kuijk C and Nevitt MC. Vertebral fracture assessment using a semiquantitative technique. $J$ Bone Miner Res 1993; 8(9):1137-1148.

18. Hans D, Goertzen AL, Bone microarchitecture assessed by TBS predicts osteoporotic fractures independent of bone density: the Manitoba study. Bone Miner Res 2011; 26(11):2762-2769. 